\title{
Stable coronary artery disease: clinical case analysis according to esc and ACC/AHA guidelines (ESC 2013, ACC/AHA 2012 and focused update 2014)
}

\author{
Danijela Trifunović-Zamaklar ${ }^{1,2}$, Nikola Bošković ${ }^{1}$, Vojislav Giga ${ }^{1,2}$, Ida Rakočević ${ }^{1}$ \\ Milan T. Petrovići, ${ }^{1,2}$ Ivana Nedeljković ${ }^{1,2}$, Dejan Orlić ${ }^{1,2}$, Branko Beleslin ${ }^{1,2}$, \\ AnaĐorđević-Dikić ${ }^{1,2}$, Jelena Stepanović1,2. \\ ${ }^{1}$ Cardiology Clinic, Clinical Centre of Serbia, Belgrade, Serbia, ${ }^{2}$ School of Medicine, Belgrade University, Belgrade, Serbia
}

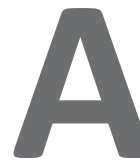

47 years old patient, with no previous history of heart disease, presented to the cardiologist with effort chest pain. He is ex-smoker, with hypertension grade $I$ and has combined hyperlipoproteinemia (total cholesterol $6.7 \mathrm{mmol} / \mathrm{l}$, LDL-Hol $4.1 \mathrm{mmol} / \mathrm{l}$, HDL $0.8 \mathrm{mmol} / \mathrm{I}$ and TG $2.8 \mathrm{mmol} / \mathrm{I})$. The patient was without therapy, but after initial medical examination, the doctor prescribed the following treatment: Acetyl-salicilic acid 1 x $100 \mathrm{mg}$, Nebivolol 1 x $2.5 \mathrm{mg}$, Ramipril $1 \times 1.25 \mathrm{mg}$, Isosorbide mono-nitrate $20 \mathrm{mg}+20 \mathrm{mg}+0$, Pravastatin $1 \times 20 \mathrm{mg}$ and Fenofibrate $1 \times 160 \mathrm{mg}$. The patient was referred to the echocardiography and stress test. Echocardiography (30.06.2011.) revealed normal left ventricle diameters (5.4 / $3.2 \mathrm{~cm}$ ), preserved global systolic function (EF $70 \%)$, without segmental wall motion abnormalities and with preserved diastolic function. The treadmill exercise test according to Bruce protocol (30.11.2011.) was stopped in the first minute of the fourth stage, due to the patient's fatigue and mild chest pain. One millimeter ST-segment depression in leads D2, D3, aVF and V4-V6 occurred during the test and recovery period. Based on these results the patient was referred to coronary angiography. Coronary angiography (30.08.2011.) showed mid LAD stenosis up to $50 \%$, distal LAD stenosis of 70 $90 \%, 70-90 \%$ ostial stenosis of the first diagonal branch, distal Cx narrowing of $70 \%$ and $90-99 \%$ stenosis of the proximal RCA. Syntax score was 19. PCl with stent implantation in proximal RCA was attempted during the procedure, but without success. Based on these findings it was decided to refer patient to stress echocardiography test (SEHO) in order to determine the extent and time of onset of myocardial ischemia. SEHO test was done under the full medical therapy (26.09.2011.) and stopped in the first minute of the IV stage, without chest pain, clinical, electrocardiographic and echocardiographic signs of reduced coronary reserve. The patient had Duke score of 10, functional capacity of 11 MET and reached submaximal frequency (SMF). His heart rate recovery (HRR) was appropriate ( $42 / \mathrm{min})$. Based on these results and high degree of workload achieved without clear signs of myocardial ischemia, it was decided to leave the patient on optimal medical therapy (OMT) and schedule him for the control SEHO tests. The control SEHO tests were done annually and the last (16.03.2016.) was still negative for ischemia at the same workload. Prognostic parameters were similar compared to the SEHO results from 26.09.2011: Duke score 10, functional capacity $11 \mathrm{MET}$, sub maximal frequency (SMF) was reached and the recovery of cardiac frequency (HRR) was appropriate. During the follow-up period the patient was asymptomatic.

\section{The therapeutic challenges and management dilemmas}

The patient from our case is a typical patient with stable coronary artery disease (SCAD). The basic dilemma regarding the management of these patients is whether optimal medical therapy (OMT) alone is sufficient or the patient should be referred to the invasive coronary angiography (ICA) and, if necessary, to myocardial revascularization. The essential question is which of these two therapeutic approaches, OMT or revascularization, has better effect on the quality of life and survival?

\section{What do the guidelines say?}

Ischemic heart disease (IHD) is the most common cause of mortality and morbidity in developed and developing countries ${ }^{1}$. Today, there are numerous scores and tables helping us to determine, with great certainty, the probability of having IHD, and further on to perform risk stratification based on clinical data and results of noninvasive diagnostic methods ${ }^{2-5}$. Standard treatments for IHD is OMT, risk factors control with life style modification and, when needed, invasive treatment (percutaneous coronary interventions - $\mathrm{PCl}$ or coronary artery bypass grafting-CABG). In the recent years invasive management with $\mathrm{PCl}$ and/or CABG is frequently preferred over OMT alone. However, although progression and development 
of invasive technique for IHD are impressive in the last decade, up to now, we do not have clear, strong and convincing evidence based data from clinical trials in favors of invasive IHD treatment over the OMT alone ${ }^{3-8}$.

Guidelines for SCAD were issued by European Society of cardiology (ESC) in 20134, and by American College of Cardiology/American Heart Association (ACC/AHA) in 2012 and updated version in 2014 ${ }^{3,5}$.

\section{Approach to the patient with suspected stable coronary artery disease}

Guidelines for the management of SCAD issued by European Society of Cardiology (ESC) in 2013 proposed three-step approach for the management of patient with chest pain and suspect SCAD ${ }^{(4)}$. The first step is to determine pre-test probability (PTP), clinical judgment that the patient has SCAD. In the second step, patients with intermediate PTP for SCAD should be referred to non-invasive testing in order to confirm or rule out the SCAD diagnosis. In the third step, a patient with a confirmed diagnosis of SCAD is given OMT and risk of adverse coronary events (so call event risk) is assessed based on the results of available non-invasive tests. The aim is to identify those patients who may benefit from invasive diagnostics (coronary angiography) and revascularization. According to the same guidelines, depending on the severity of symptoms, the patient can undergo early coronary angiography with, if necessary, invasive confirmation of the significant stenosis (using FFR), followed by revascularization, thus skipping steps 2 and 3.

\section{Determination of pretest probability in patients with chest pain}

In clinical practice, the most used is is Diamond Forrester table (modified by Tessa Genders et al. 2011.) to determine the pre-test probability of the SCAD based on the nature of chest pain, sex and age. ${ }^{(9)}$ Typical anginal pain has the following characteristics: (1) retrosternal localization with characteristic quality and duration; (2) it is provoked by physical exertion or emotional stress; (3) it is stopped with the rest or nitroglycerin application. Atypical chest pain has two of the given characteristics, and non cardial pain has one or none of the following characteristics. This table is Table 13 is the 2013 ESC recommendations for the diagnosis and treatment of SCAD and is identical to the table from the 2012 ACC/AHA guidelines for SCAD. Using this table we can divide the patients with the chest pain into three categories: patients with low pretest probability (PTP) $<15 \%$, patients with intermediate PTP (16-85\%) and patients with high PTP $(>85 \%)^{(4)}$.

According to the 2013 ESC recommendations for SCAD, patients with low pre-test probability $(<15 \%)$ do not require testing; patients with intermediate pre-test probability (15-65\%) and LVEF $\geq 50 \%$, should be referred to the stress ECG as the initial test, but if stress imaging test available, it is more desirable (SEHO, MRI, SPECT, PET); patients with high PTP (66-85\%) and LVEF $<50 \%$ even without typical angina symptoms, should go directly to stress imaging test (SEHO, CMR, SPECT, PET); for patients with very high PTP (over $85 \%$ ) it can be considered that SCAD already exists. For them it is not necessary to do diagnostic tests, and coronary angiography without noninvasive testing is indicated. Tests can be done for risk stratification. In these recommendations coronary CTA may be considered as a first noninvasive test in patients with intermediate PTP (15-50\%) if the patient is suitable for the test and if adequate technology and local expertise are available. Also, coronary CTA (computed tomography angiography) is recommended if the results of the ECG stress test or stres imaging tests are unclear. This is represented as Figure 2 in the ESC guidelines ${ }^{(4)}$.

According to ACC/AHA recommendations for SCAD from 2012 and updated version from 2014, patients with intermediate PTP (15-65\%) should be addressed to the stress ECG test, if the ECG at rest is interpretable, independently of LVEF. If the ECG at rest is not interpretable (LBBB, pacemakers, WPW syndrome, ST segment depression), patients should be referred to stress imaging test (SPECT, SEHO test, pharmacological CMR). Also, patients with high-intermediate pre-test probability (66-85\%) should be refered to the imaging stress test (SPECT, SEHO test, pharmacological CMR), just like in the ESC guidelines. Patients whose ECG is not interpretable in rest or who have had previous myocardial revascularization sould be refered to the pharmalogical myocardial perfusion stress test or stress ECHO test. Patients who are not able to exercise should be referred to the pharmalogical myocardial perfusion stress test, stress ECHO test or coronary CTA. Also patients who have contraindications to stress testing should be referred to coronary CTA. This is represented as Figure 2 in the ACC/AHA guidelines ${ }^{(3)}$.

\section{ESC and ACC/AHA guidelines: how to manage our patient?}

According to Diamond-Forrester table, our patient had $69 \%$ PTP for SCAD. Since he did not have previous CVD, and his ECG in rest was interpretable and EF $>50 \%$, the decision to refer him to stress test was in accordance with the current guidelines. Based on the positive stress test, the patient was referred to coronary angiography, as also suggested by the guidelines.

\section{Risk stratification for the adverse cardio- vascular events}

Non-invasive tests are used not only for diagnosing SCAD, but also to assess risk for adverse cardiovascular events (cardiovascular death or myocardial infarction). The risk is usually expressed as an annual probability of myocardial infarction or death and is defined as low (annual probability of less than $1 \%$ ), medium (annual probability of 1-3\%) and high risk (annual probability of $>3 \%$ ). Risk stratification of patients with SCAD can be done using various non-invasive tests (Table 17 in the ESC guidelines and Table 14 in theACC/ AHA guidelines) $(3,4)$. In the ESC guidelines if the patient referred to the ECG stress testing and had a ST depression $\geq 2 \mathrm{~mm}$ he/she has a high risk, if 
the ST depression was $1-2 \mathrm{~mm}$ it is intermediate risk and if there were no ST changes and no chest pain then it is low risk. For the patient referred to stress imaging tests the risk is defined as high in case of area of ischaemia $>10 \%$ (>10\% for SPECT; limited quantitative data for CMR - probably $\geq 2 / 16$ segments with new perfusion defects or $\geq 3$ dobutamine-induced dysfunctional segments; $\geq 3$ segments of LV by stress echo); intermediate risk is defined if area of ischaemia is between 1 to $10 \%$ or any ischaemia less than high risk by CMR or stress echo; low risk is considered in case of no ischemia on the test. If the patient referred to the coronary CTA, the risk is high in case of significant high risk lesion (three-vessels disease with proximal stenoses, LM, and proximal anterior descending CAD), the risk is intermediate in case of significant lesion(s) in large and proximal coronary artery(ies) and the risk is low in caseof normal coronary arteries or presence of plaques only.

$\mathrm{ACC} / \mathrm{AHA}$ guidelines are very similar regarding this issue and $A H A$ recommendations also includes Agatston calcium score. In these guidelines patients with CAC score is $>400$ Agatston units are in the high risk, patients with CAC score between 100 and 399 Agatston units have intermediate risk and patients with CAC score $<100$ Agatston units have low risk.

According to ESC guidelines, prognostic markers useful for risk stratification during ECG stress test (ergometry) are: functional capacity (MET - Metabolic Equivalent of Task), i.e. exercise capacity, blood pressure changes during the test and exercise induced myocardial ischaemia based on clinical and ECG parameters. From all of these prognostic markers, functional capacity has the strongest relation with adverse CV events and mortality, independent of age, gender and the presence and severity of coronary artery disease. ${ }^{10,11}$ Functional capacity can be expressed as the maximum duration of exercise, maximal achieved metabolic equivalent (1 MET $=3.5 \mathrm{ml} \mathrm{O2} / \mathrm{kg} /$ min), maximal workload (expressed in watts), maximal heart rate and the so-called "double product" (blood pressure $x$ heart rate). Duke treadmill score is well-validated risks score that combines treadmill exercise time (in minutes), maximum net ST segment deviation during the test (in $\mathrm{mm}$ ) and exercise induced angina: Duke treadmill score $=$ exercise time $-5 x S T$ deviation- $4 x$ angina index. According to Duke risk score the risk can be low (scor $\geq 5)$, medium $(-10 \leq$ skor $<5)$ and high (score $<-10) .{ }^{12,13}$ Heart Rate recovery (HRR) is a predictor of mortality, independent of the angiographic severity of coronary artery disease $(\mathrm{HRR}=$ maximal $\mathrm{HR}$ during exercise $-\mathrm{HR} 1 \mathrm{~min}$ after exercise; abnormal (inadequate) heart rate recovery is $\leq 18$ beats per minute). ${ }^{14,15}$

\section{Management of the stable coronary artery disease}

According to the ESC guidelines, treatment options for patients with SCAD are based on risk stratification (Figure 3 in the ESC guidelines) and can help in deciding whether a patient should be referred to, in addition to OMT and invasive coronary angiography (ICA), followed by angiographic findings and myocardial revascularization. If the patients have a low event risk they should be on optimal medicament treatment (OMT). If the symptoms are improved continue OMT, if not the medical treatment should be intensified. If the patients have symptoms even after intensifying the medical treatment, refer the patient to the ICA. In patients with intermediate event risk start administering OMT in the same way as in the patients with low risk but ICA may be considered based on the co-morbidities and patient's preference. Patients with high event risk should be referred to ICA. ${ }^{4}$

\section{Risk factors control and pharmacological management of the stable coronary artery disease}

It has been shown that optimal medical therapy (which includes the optimal way of life with the achievement of target lipid levels, blood pressure, and glucose in the blood) have the same prognostic, and symptomatic character as revascularization, if reversible ischemia induced by the ischemia provocation tests does not involve more than $10 \%$ of the left ventricular myocardium (which corresponds to echocardiographic deterioration of kinetics in at least two segments).

Regardless of the chosen management, pharmacological treatment or invasive methods, the first-line therapy in all patients should be risk factors control (smoking cessation, dietary intake, control of the blood lipids level, hypertension and diabetes) as well as the lifestyle modification (moderate physical activity, regulation of body weight, etc.). ESC and ACC/AHA guidelines in this regard are very similar, but the ACC/AHA guidelines provide much more information about the education of doctors on the approach to patients, as well as different methods of education patients themselves and methods of counseling (education via the Internet, group counseling, etc). Also ACC/AHA guidelines, as opposed to the ESC guidelines, suggest different ways of patient's monitoring. ${ }^{8}$

Depending on the symptoms, the functional and anatomical complexities, stable angina pectoris can be treated with optimal medical therapy and/or revascularization.

The goals of optimal medical therapy are to eliminate or reduce angina and to prevent adverse cardiovascular events.

Myocardial ischaemia occurs due to the mismatch between myocardial oxygen supply and myocardial needs, as defined by the "double product" (heart rate $x$ systolic blood pressure). For this reason, any antianginal drugs are aimed to reduce the consumption of oxygen by the myocardium (by reducing heart rate and/or blood pressure: beta blockers, non-dihydropyridine calcium channel blockers, all antihypertensives, vasodilators). In therapy of vasospastic angina (Prinzmetal) vasodilators are essential (long and short-acting nitrates and calcium channel blockers).

Pharmacological treatment should be administered to all patients, regardless of whether they were treated invasively or not, and the ESC and ACC/AHA guidelines are identical. The first-line treatment of symptoms in patients with suspected or proven SCAD includes: short-acting 
nitrates in combination with $\beta$-blockers or calcium channel blockers in case of intolerance /contraindication for the use of $\beta$-blockers or in combination if CCS $>2$. Several classes of drugs have been proved to have essential impact on the progression of coronary atherosclerosis and atherothrombosis, consequently influence symptoms of IHD, but more importantly, prevent adverse cardiovascular event and modify prognosis. These drugs are: antiplatelet drugs (primarily aspirin), statins, ACE inhibitors and angiotensin receptor blockers. If symptom control in patients with SCAD is not satisfactory, introduction of the one of the second line drugs is needed: long-acting nitrates, trimetazidine, ranolazine, nicorandil or ivabradine.

Pharmacological treatment of SCAD according to the ESC guidelines is shown as Figure 4. and there is no difference in the therapeutic algorithm compared to the ACC/AHA guidelines. ${ }^{3,4}$

CASS (Coronary artery surgery study) study included 780 patients with stable angina pectoris class I or II of the Canadian Association of Cardiovascular Diseases (CCS), who had angiographically verified $\geq 70 \%$ stenosis of the LAD, RCA or Cx or $\geq 50 \%$ stenosis of the proximal LAD or LM, and who had a previous CABG or unstable angina. This study compared the results of CABG + OMT vs OMT only. Patients were followed for an average of 46 months. The results showed that there was no significant difference in mortality between the two groups, regardless of the number of diseased vessels. The annual mortality rate in patients with one, two and threevessels disease who were treated with the combined therapy (CABG + OMT ) was $0.7 \%, 1 \%$ and $1.5 \%$ and in patients only on OMT was $1.4 \%, 2.1 \%$ and $2.5 \% .^{6}$

The Clinical Outcomes Utilizing Revascularization and Aggressive Drug Evaluation (COURAGE) trial $(n=2287$ ) compared $\mathrm{PCl}+\mathrm{OMT}$ with OMT only, in patients with SCAD or ischaemia and coronary lesions suitable for $\mathrm{PCl}$. The target study population for the COURAGE trial included patients with chronic angina pectoris Canadian Cardiovascular Society (CCS) Class I-III, stable post-MI patients and asymptomatic patients with objective evidence of myocardial ischaemia. All patients had angiographically defined CAD, with at least one vessel meeting AHA/American College of Cardiology (ACC) Class I or II indications for $\mathrm{PCl}$. Patients with a prior CABG were accepted. Patients with stenosis $>80 \%$ in one or more vessels, supplying the large area of the myocardium, could be enrolled even in the absence of objective ischaemia. The primary endpoint, all-cause death or non-fatal MI, did not differ between the two groups during a mean follow-up of 4.6 years. However, in patients who were invasively treated, freedom from angina was significantly better up to 3 years of follow-up. In a sub-study, patients with $>10 \%$ ischaemia on stress myocardial perfusion scintigraphy had a higher rate of death or MI. More $\mathrm{PCl}+\mathrm{OMT}$ patients exhibited significant ischaemia reduction (33 vs. $19 \% ; P=0.0004)$. Patients with ischaemia reduction had lower unadjusted risk for death or MI, particularly if baseline ischaemia was moderate to severe ${ }^{7}$.

COURAGE II study, a 15-year follow-up of 1,211 patients patients included in the original study, also showed no difference in mortality and incidence of non-fatal MI between patients with $\mathrm{PCl}+\mathrm{OMT}$ vs OMT (HR 0.95: $95 \% \mathrm{Cl} 0,79$ to $1.13, \mathrm{p}=0.53$ ). ${ }^{8}$

The ISCHEMIA study is currently in progress (International Study of Comparative Health Effectiveness with Medical and Invasive Approaches. It is aimed to define the role of an invasive approach in patients with stable ischemic heart disease (SIHD) and substantial ischemia. The trial hypothesis is that cardiac catheterization followed by complete revascularization plus optimal medical therapy (OMT) is superior to OMT alone, for patients with moderate-severe ischemia on stress imaging. The primary endpoint will be time to cardiovascular death, myocardial infarction (MI), or hospitalization for unstable angina, resuscitated cardiac arrest, or heart failure. The hypothesis that the invasive strategy will improve quality of life will also be tested. Cost-effectiveness will be assessed. Observational data suggest that revascularization of patients with moderate-severe ischemia is associated with a lower likelihood of death and MI; this was not observed in patients with lesser degrees of ischemia. Only about half of patients with moderate-severe ischemia are referred for catheterization. It is unknown whether used rates for catheterization and revascularization are appropriate for optimal patient management in the era of modern medical therapy (particularly with high dose statins and antiplatelet therapy). This issue cannot be resolved using available data, because prior clinical trials regarding SIHD have enrolled patients after catheterization, at which point there is substantial selection bias for the enrollment based on coronary anatomy. Given the potential for improved survival and fewer cardiac events, as a result of revascularization and the significant expense and risks associated with invasive management, the role of an invasive strategy is critically important to define. The proposed ISCHEMIA trial will be a prospective, multicenter, international, randomized, controlled trial that will directly address the need for an invasive strategy-cath and revascularization-in patients with SIHD. A total of 8,000 patients with moderate-severe ischemia and left ventricular ejection fraction $>35 \%$ will be enrolled after stress imaging from more than 400 sites. Based on the need to exclude significant left main coronary artery disease, patients who meet eligibility criteria will undergo blinded coronary CT angiography. Patients will be randomized to an invasive group that will undergo routine cath with optimal revascularization, if feasible, plus OMT or to a group that receives OMT alone (http://grantome.com/grant/NIH/ U01-HL105907-03 ) $)^{16}$.

However, none of these studies took into account the prognostic markers of exercise testing such as functional capacity, heart rate recovery, blood pressure response, Duke treadmill score, which proved to be good predictors of morbidity and mortality of cardiovascular disease $\mathrm{e}^{15}$. The study of the Myers et al. compared the effect of physical activity (expressed as a MET-tested load) on the mortality of any cause among patients who did not and those who had a previous history of cardiovascular disease. This study included 6213 men who were followed for $6.2 \pm 3.7$ years. It turned out that the greater achieved MET reduces the risk of dying in both populations ${ }^{10}$. Patients were 
divided into two groups: 3679 with a positive test or previous coronary artery disease and 2534 patients with a normal test and without coronary artery disease. After the test all patients were divided in the five groups (quintiles of exercise capacity) according to the achieved MET: les than 5 MET, 6-7.9 MET, 8-9.9 MET, 10-12.9 MET and those who achieved 13 MET or higher. The group of patients who achieved 13 MET or higher was used as a reference group. It has been showed that the relative risk of death was the highest among the groups who achieved less than 5 MET (2.95-6.83 among healthy subjects and 3.29-5.16 among the patients with previous history of CAD, $p=n s$ ) and gradually decreased among the groups with the smallest value among the patients who achieved 10-12.9 MET (0.68-2.22 2.95-6.83 among healthy subjects and 1.352.19 among the patients with previous history of CAD, $p=$ $n s)$. In both groups, in healthy and in patients with coronary artery disease, achieved maximum functional capacity was a stronger independent predictor of increased risk of mortality compared to the known risk factors such as hypertension, smoking, diabetes as well as other prognostic test parameters, including ST segment depression, and the maximum achieved heart rate during the test or the occurrence of the arrhythmias during the test.

According to non-invasive risk stratification our patient had low probability for the occurrence of myocardial infarction or death. In concordance with current guidelines OMT was initiated, relieving the symptoms, therefore further follow up is recommended.

\section{Conclusion}

Early diagnosis and appropriate treatment of coronary artery disease is one of the greatest challenges in modern cardiology. Despite major breakthrough in noninvasive and invasive diagnostic procedure and modern treatment options, CAD still has high morbidity and mortality. Since there is no solid evidence to show the advantages of the invasive treatment compared to OMT, it is necessary to have an individual approach for each patient. Studies such as COURAGE I and II, CASS and the study of Myers et al., as well as our patient from the case report showed that besides the test results prognostic parameters of the test, especally functional capacity (expressed through MET), Duke treadmill score, achieved target heart rate and heart rate recovery have great importance. These studies also suggest that regardless of the therapeutic mode (medical or invasive therapy), risk factors control and lifestyle modifications are the first and necessary steps when treating these patients and highlight the importance of primary and secondary prevention of coronary artery disease.

\section{References}

1. Crea F. Chronic isheamic heart disease. In ESC Textbook of cardiologOxford: Oxford University Press; 2010.

2. Diamond GA. A clinically relevant classification of chest discomfort. J Am Coll Cardiol. 1983;1(2 Pt 1):574-5.

3. Fihn SD, Gardin JM, Abrams J, Berra K, Blankenship JC, Dallas AP, et al. 2012 ACCF/AHA/ACP/AATS/PCNA/SCAI/STS guideline for the diagnosis and management of patients with stable ischemic heart disease: a report of the American College of Cardiology Foundation/ American Heart Association task force on practice guidelines, and the American College of Physicians, American Association for Thoracic Surgery, Preventive Cardiovascular Nurses Association, Society for Cardiovascular Angiography and Interventions, and Society of Thoracic Surgeons. Circulation. 2012;126(25):e354-471.

4. Montalescot G, Sechtem U, Achenbach S, Andreotti F, Arden C, Budaj A, et al. 2013 ESC guidelines on the management of stable coronary artery disease: the Task Force on the management of stable coronary artery disease of the European Society of Cardiology. Eur Heart J. 2013;34(38):2949-3003.

5. Fihn SD, Blankenship JC, Alexander KP, Bittl JA, Byrne JG, Fletcher BJ, et al. 2014 ACC/AHA/AATS/PCNA/SCAI/STS focused update of the guideline for the diagnosis and management of patients with stable ischemic heart disease: a report of the American College of Cardiology/American Heart Association Task Force on Practice Guidelines, and the American Association for Thoracic Surgery, Preventive Cardiovascular Nurses Association, Society for Cardiovascular Angiography and Interventions, and Society of Thoracic Surgeons. J Am Coll Cardiol. 2014;64(18):1929-49.

6. Coronary artery surgery study (CASS): a randomized trial of coronary artery bypass surgery. Survival data. Circulation. 1983;68(5):939-50.

7. Boden WE, O'Rourke RA, Teo KK, Hartigan PM, Maron DJ, Kostuk WJ, et al. Optimal medical therapy with or without $\mathrm{PCI}$ for stable coronary disease. N Eng J Med. 2007;356(15):1503-16.

8. Sedlis SP, Hartigan PM, Teo KK, Maron DJ, Spertus JA, Mancini GB, et al. Effect of PCl on Long-Term Survival in Patients with Stable Ischemic Heart Disease. N Eng J Med. 2015;373(20):1937-46.

9. Genders TS, Steyerberg EW, Alkadhi H, Leschka S, Desbiolles L, Nieman K, et al. A clinical prediction rule for the diagnosis of coronary artery disease: validation, updating, and extension. Eur Heart J. 2011;32(11):1316-30.

10. Myers J, Prakash M, Froelicher V, Do D, Partington S, Atwood JE. Exercise capacity and mortality among men referred for exercise testing. N Eng J Med. 2002;346(11):793-801.

11. Roger VL, Jacobsen SJ, Pellikka PA, Miller TD, Bailey KR, Gersh BJ. Prognostic value of treadmill exercise testing: a population-based study in Olmsted County, Minnesota. Circulation. 1998;98(25):2836-41.

12. Mark DB, Hlatky MA, Harrell FE, Jr., Lee KL, Califf RM, Pryor DB. Exercise treadmill score for predicting prognosis in coronary artery disease. Annals of internal medicine. 1987;106(6):793-800.

13. Mark DB, Shaw L, Harrell FE, Jr., Hlatky MA, Lee KL, Bengtson JR, et al. Prognostic value of a treadmill exercise score in outpatients with suspected coronary artery disease. N Eng J Med. 1991;325(12):849-53.

14. Cole CR, Blackstone EH, Pashkow FJ, Snader CE, Lauer MS. Heartrate recovery immediately after exercise as a predictor of mortality. N Eng J Med. 1999;341(18):1351-7.

15. Vivekananthan DP, Blackstone EH, Pothier CE, Lauer MS. Heart rate recovery after exercise is a predictor of mortality, independent of the angiographic severity of coronary disease. J Am Coll Cardiol. 2003;42(5):831-8.

16. http://grantome.com/grant/NIH/U01-HL105907-03 